\title{
LHCb Software and Conditions Database Cross-Compatibility Tracking System: a Graph-Theory Approach
}

\author{
M. Cattaneo, M. Clemencic, and I. Shapoval
}

\begin{abstract}
The Conditions Database (CondDB) of the LHCb experiment provides versioned, time dependent geometry and conditions data for all $\mathrm{LHCb}$ data processing applications (simulation, high level trigger, reconstruction, analysis). The evolution of CondDB and of the LHCb applications is a weaklyhomomorphic process. It means that compatibility between a CondDB state and LHCb application state may not be preserved across different database and application generations. More over, a CondDB state by itself belongs to a complex three-dimensional phase space which evolves according to certain CondDB selfcompatibility criteria, so it is sometimes difficult even to determine a self-consistent CondDB state. These compatibility issues may lead to various kinds of problems in the $\mathrm{LHCb}$ production, varying from unexpected application crashes to incorrect data processing results. Thus, there is a need for defining a well-established set of compatibility criteria between mentioned above entities, together with developing a compatibility tracking system which will help to discover incompatibilities avoiding their negative consequences. This paper describes an attempt to approach this goal in the frame of the graph theory which allows to formalize elegantly the task of compatibility tracking.
\end{abstract}

\section{INTRODUCTION}

I $\mathrm{N}$ the current LHCb [1] computing model [2] compatibilities of $\mathrm{LHCb}$ data processing applications and $\mathrm{LHCb}$ CondDB [3] states are not tracked and are managed in a manual and very limited way. This may lead to a diverse set of application execution failures in the LHCb production when LHCb software evolution passes non-homomorphic mutation phases. Even this phenomenon may seem to be very annoying by itself, and it is such in the LHCb collaboration running into a thousand of members. But, unfortunately, it is not the worst scenario: a danger always exist that a wrong (but not leading to application execution failure) combination of CondDB and application states may be used in production in which case the results of the LHCb application execution might not be entirely correct. This paper describes a prototype of a system that will help to track and perform automatic verification of mentioned compatibilities reliably.

We start out with an introduction of objects between which the compatibility will be considered (Section II), then, fol-

Manuscript received November 15, 2012.

M. Cattaneo is with the European Organization for Nuclear Research, Geneva, CERN CH-1211, Switzerland (e-mail: Marco.Cattaneo@cern.ch).

M. Clemencic is with the European Organization for Nuclear Research, Geneva, CERN CH-1211, Switzerland (e-mail: Marco.Clemencic@cern.ch).

I. Shapoval is with the European Organization for Nuclear Research, Geneva, CERN CH-1211, Switzerland and with the Kharkiv Institute of Physics and Technology, Kharkiv, UA-61108, Ukraine (e-mail: Illya.Shapoval@cern.ch). lowing a definition of the compatibility criteria (Section III), we will describe a design of the compatibility tracking system (Section IV).

\section{Compatibility Entities}

All compatibility entities of our consideration can be subdivided into three classes: the LHCb application states, the LHCb Conditions Database states and the physics data states. Let's now briefly describe each class.

\section{A. Conditions database entities}

A general CondDB state is fully defined by sub-states of its three partitions. So the role of the CondDB compatibility entity is given to this partition state. The partitions considered are:

- DDDB: LHCb time independent nominal detector description partition. It is used to describe the geometry of the $\mathrm{LHCb}$ detector;

- LHCBCOND/SIMCOND: LHCb detector conditions partitions. They are used to describe time dependent conditions like calibrations, alignment, etc. The former partition is used for real data reconstruction and analysis, and the latter one for experiment's detector simulation;

- DQFLAGS: LHCb data quality flags partition. It is used for tracking the quality of physics data taken by the experiment.

Each partition state is versioned: it is tagged at specific moments of the partition's evolution. The tag can be global or local, depending on whether it tags complete or partial state. The local tags play a secondary role in CondDB configuration and are usually used for CondDB fine-tuning. While a triplet of global tags, each of which corresponds to one of the three partitions mentioned above, are always used to fully define the CondDB state. Not every triplet of these tags forms a selfconsistent CondDB state (see Section III below).

\section{B. Application entities}

Each LHCb application is a client of CondDB. Some examples of LHCb applications are as follows:

- Moore - used for high level triggering;

- Brunel - used for event reconstruction;

- DaVinci - used for event selection and data analysis;

- Gauss - used for event generation and detector simulation; 
For our consideration the concrete application flavor is not important since there is no conceptual difference between them from the point of view of compatibility with a CondDB state. But what is important is that the LHCb computing model forces each application to be versioned and the version number will uniquely identify the application entity in our consideration.

\section{Physics data entities}

The raw physics data taken by the LHCb experiment passes several phases of processing by the applications mentioned in the previous subsection. Each data transformation is accomplished using a particular CondDB state (tag) and application state (version) which are stored together with processed data. So we say that each LHCb data entity is associated with a CondDB tag and application version.

\section{COMPATIBILITY RELATIONS}

Before going any further we have to specify what stands behind the compatibility concept in our problem. In the LHCb computing model we encounter the following categories of compatibility relations for the entities described in the previous section:

- CondDB self-compatibility

- Inside-partition tags compatibility

- Cross-partition tags compatibility

- External CondDB compatibility

- Compatibility of a tag with the LHCb application

- Compatibility of a tag with physics data

So let's consider all the compatibility categories in more detail.

\section{A. Conditions database self-compatibility}

One should note that the case of inside-partition compatibility (i.e. the compatibility between the tags of the same CondDB partition) has two occurrences in our problem:

- inside-partition compatibility of global CondDB states (global tags);

- inside-partition compatibility of local CondDB partition states (local tags).

The local CondDB states play a secondary role in $\mathrm{LHCb}$ applications execution so we will not consider in this paper the compatibility flavor mentioned in the latter bullet. The global CondDB state in turn, described in Section II, is a player on both the cross-partition and inside-partition tags compatibility field. However, we will not establish explicit compatibility relations within a partition since, as it will become clear in Section IV, the design of the compatibility tracking system makes this detail redundant. Let's now consider how does the cross-partition compatibility evolve.

The compatibility relations between the CondDB tags are fully defined by the content of the CondDB partitions versioned with those tags. Suppose, we have a fully compatible set of tags. Suppose also, that we are going to create a new tag in a partition which will contain some set of changes. Depending on the type of the coming change we may obtain

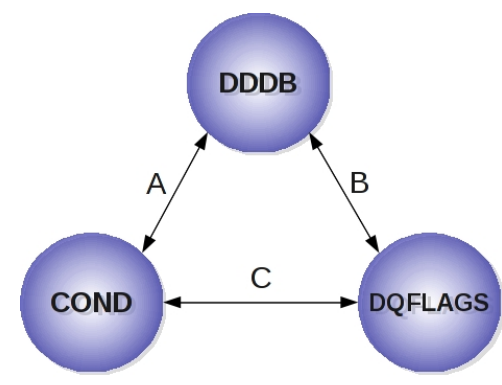

Fig. 1. CondDB partitions together with their compatibility relations. The COND notation generalizes LHCBCOND and SIMCOND partition cases, mentioned in Section II.

TABLE I

THE MAP OF SOURCES FOR THE INTERNAL CONDDB COMPATIBILITY VIOLATION. THE RELATION TYPES $A, B$ AND $C$ ARE DEFINED IN Figure 1.

\begin{tabular}{llll}
\hline & \multicolumn{3}{c}{$\begin{array}{l}\text { Potential to break certain compatibility } \\
\text { relation }\end{array}$} \\
\cline { 2 - 4 } Type of CondDB change & $\mathbf{A}$ & B & C \\
\hline $\begin{array}{l}\text { non-structural changes } \\
\text { (e.g., alignment, calibration }\end{array}$ & No & No & No \\
or particle table updates) & & No & No \\
\hline $\begin{array}{l}\text { structural changes (e.g., } \\
\text { new detector or condition } \\
\text { elements, new database ob- } \\
\text { jects' dependencies) }\end{array}$ & & & \\
\hline
\end{tabular}

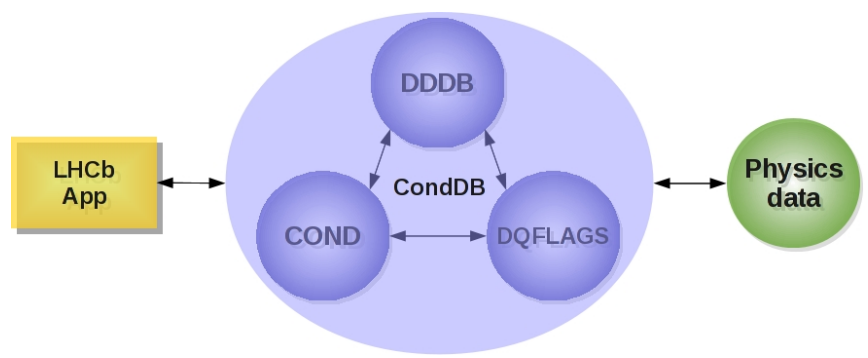

Fig. 2. More complete view (relatively to Figure 1) of the compatibility relations topology: the $\mathrm{LHCb}$ application and physics data entities are added.

the new tag either compatible, or not, with the previous tags. Figure 1 and Table I are summarizing two common cases showing the compatibility implications of adding new changes to CondDB. Declaring the compatibilities of a new tag with the previous ones is a responsibility of the LHCb sub-detector groups.

\section{B. External Conditions database compatibility}

We say that a CondDB tag, say tag T, is compatible with the LHCb application and a data entity (see Figure 2) if the following three conditions are met:

- The LHCb application does not fail while executing over the CondDB of a version identified by the tag $\mathrm{T}$;

- The CondDB structure expected by the LHCb application maps identically to the actual CondDB structure declared by the tag $\mathrm{T}$;

- The tag, associated with a data, is equal to the tag T.

Most of the failures of the first bullet type are caused by the second bullet condition violation. 


\section{COMPATIBILITY TRACKING SYSTEM}

The compatibility tracking system is aimed to perform the following tasks:

- automatic verification of both internal and external (see Section III) CondDB compatibilities:

- at application configuration stage;

- at events processing stage;

- automatic derivation of compatible CondDB state for specific LHCb application, physics data and user criteria:

- full autonomous derivation of compatible CondDB state (e.g. by data type, or by time);

- tuned derivation of compatible CondDB state based on user's constraints (e.g., if CondDB state is specified by user partially: a subset of tags only is given);

- easy exploration of the internal and external compatibility relations' trees (e.g., through the web interface).

The first bullet's sub-item will solve most of the compatibility problems since currently most of them come from wrong application configuration. The second sub-item should provide a deeper compatibility verification level for special cases when the LHCb application meets events processed using tags different from those declared by the application configuration. The second bullet will give an option of smart and silent compatible tags setup for a given application by a set of user constraints. And the last bullet will serve mostly the purposes of more convenient and reliable CondDB management for the CondDB sub-detector support groups and for CondDB managers.

In the following subsection we will see how all mentioned tasks can be processed within a generic approach.

\section{A. Handling all compatibility categories in a generic way}

Now that the nature of all compatibility entities and relations between them are described (Sections II and III) we can put the problem on a higher abstraction level. The complete compatibility relations tree can be expressed in the frame of graph theory in terms of a directed acyclic weighted attributed graph (DAWAG). We can define the graph elements in the following way:

- A node: a compatibility entity (either an application, or a tag)

- An application node per each application version

- A tag node fully identified by tag name, CondDB partition, data type

- An edge: compatibility relation (either between application and a tag node, or between two tag nodes)

Note that for our purposes the graph must be weighted - we assign to each edge a numerical value that will represent a sequence number of the compatibility relation inserted for that node. This will enable the compatibility history tracking and the possibility, for example, of fast search for the latest compatible tags. The graph must be also attributed to store extra information about the compatibility entities:

- for an application node: application name, application version, release date;

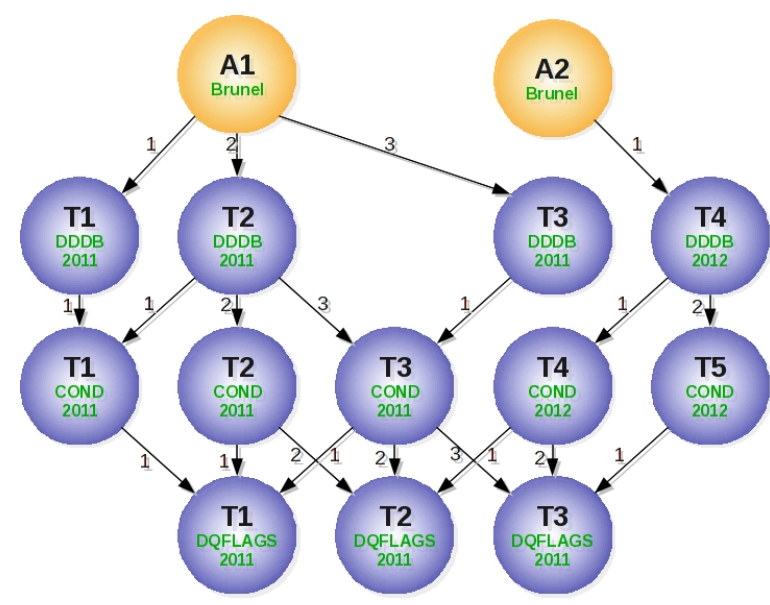

Fig. 3. An example of a DAWA graph for Brunel application. The A-nodes represent Brunel versions while the T-nodes - the CondDB tags entities. The green text of each node represents some of the attributes (CondDB partition name field (DDDB, COND, DQFLAGS), a data type field $(2011,2012)$.

- for a tag node: tag name, CondDB partition, tag's release date, physics data type the tag is created for, tag's hash sum.

An example of such a graph is shown in Figure 3. Such representation will enable the extraction of the compatibility information in a natural way. In such approach the procedure narrows down to searching for the graph path which meets conditions of a query. The graph path will always start at an application family node which will play a broker role in almost all usage scenarios (see Figure 3) and will have a length of five nodes (an application family node, versioned application instance plus one tag per each CondDB partition). One can imagine some of the possible queries which can be extracted from the graph:

- derive the full set of the latest compatible tags for an application of a specific version and for a specific data type;

- check that a set of entities is compatible (tags; tags and an application);

- extract all possible entities that can augment the requested ones to a full compatible path.

\section{B. Overview of the compatibility tracking system}

To process the tasks listed at the beginning of this section the compatibility tracking system (CTS) will have to "talk" to a graph described in the previous subsection (see Figure 3). In particular, the system will perform two kinds of actions:

- injection of new compatibility relations to the graph;

- analysis of the graph to get a compatibility solution.

In the latter bullet by the compatibility solution we mean, depending on a user's query type, either an answer whether some set of entities is compatible, or a set of compatible entities which meet requested constraints.

A scheme of the system, able to perform these tasks, is shown in Figure 4. One can see that the system expects to receive an input which can be: 


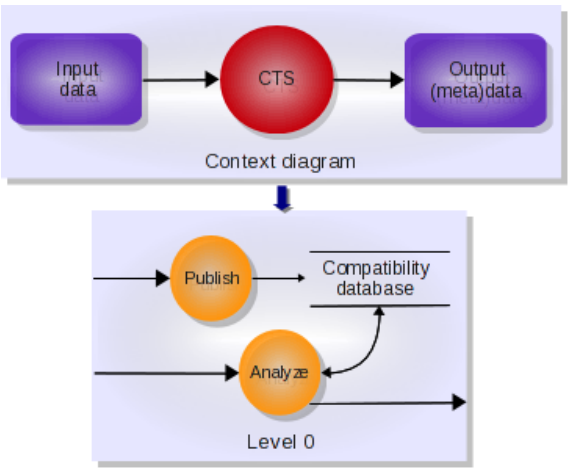

Fig. 4. Leveled data flow diagram for the compatibility tracking system (CTS) (shown in the Yourdon-DeMarco DFD notation [4]). The Level 0 layer depicts the CTS intrinsics. See Subsection IV-C for the Compatibility database description.

- new compatibility information (supplied by the CondDB Manager);

- client's query for a compatibility solution (a client can be a regular LHCb application, or a web compatibility portal agent).

The output is obviously only expected in the second input scenario described above.

\section{CTS core: a relational database versus a graph database}

A core of the compatibility tracking machinery has to be a database supporting the compatibility graph described in Subsection IV-A. The choice of a technology which can drive such database depends on several factors. The key ones are:

- how does the technology of a choice fit the general concept of a task:

- information entities to store;

- querying efficiency;

- what are the requirements of the chosen technology (available database API, database hosting options, etc.);

- technology support by manufacturer;

The most obvious database technologies to be considered in the frame of our problem in our opinion were:

- A relational one (e.g., SQLite, see [5]);

- A graph-oriented one (e.g., Neo4j NOSQL graph database, see [6]).

The Neo4j NOSQL database is one of the most advanced graph-oriented databases present on the market nowadays. Some of the main features of the Neo4j database are as follows:

- Intuitive graph-oriented model for data representation;

- True ACID transactions;

- Massive scalability (up to several billions of graph nodes);

- Native storage manager optimized for storing graph structures;

- Bindings for Python, Jython, Ruby, Clojure, Scala, JavaScript, Java, Groovy.

The graph-oriented model of Neo4j fits our goal of representing the compatibility graph with performance and helps
TABLE II

A COMPARISON OF THE SEVERAL SQLITE AND NEO4J DATABASES KEY FEATURES.

\begin{tabular}{llllll}
\hline & \multicolumn{4}{c}{ Database technology features } \\
\cline { 2 - 6 } $\begin{array}{l}\text { Database } \\
\text { technol- } \\
\text { ogy }\end{array}$ & $\begin{array}{l}\text { Field- } \\
\text { oriented }\end{array}$ & DBMS & $\begin{array}{l}\text { REST } \\
\text { API }\end{array}$ & $\begin{array}{l}\text { Deployment License } \\
\text { needed }\end{array}$ \\
\hline SQLite & No & Embedded & No & No & $\begin{array}{l}\text { Public do- } \\
\text { main }\end{array}$ \\
\hline Ne04j & Yes & $\begin{array}{l}\text { Embedded, } \\
\text { Server- } \\
\text { based }\end{array}$ & Yes & Yes, No & $\begin{array}{l}\text { GPLv3, } \\
\text { AGPLv3, } \\
\text { commercial }\end{array}$ \\
\hline
\end{tabular}

to avoid any possible aspect of the known object-relational impedance mismatch problem (see [7], [8]). Also an absence of necessity of schema specification in Neo4j makes possible CTS data model evolution an easier task. At the same time it has several drawbacks, like dual licensing (some features, like high-availability mode and runtime backups, are under commercial license) and not an oustanding set of language bindings (this, though, is compensated by the Neo4j REST API).

The SQLite does not need any promotion - it is very popular as an embedded SQL database engine with an excellent product support. Storing a graph in a relational database is possible and the SQLite engine could be an out-of-the-box solution taking into account the availability of the database engine libraries in the $\mathrm{LHCb}$ computing environment $(\mathrm{Neo} 4 \mathrm{j}$, instead, needs deployment). It has although an obvious drawback: SQLite's relational nature doesn't fit the graph-oriented spirit of our compatibility tracking system design with graphs as a tracking unit. This has the following consequences:

- increased amount and complexity of client-side code needed for relational database;

- increased execution time of client-side code due to needed graph object assembling and disassembling, or due to other relational data manipulation;

- low database querying efficiency compared to graphoriented database approach.

As mentioned in the last bullet above, querying a relational database for graph-like information, particularly traversing it, can be expensive (due to the number of potential SQL joins) in comparison with the graph database case which is naturally optimized for the graph-related queries [9].

A comparison of the features of both technologies relevant to our compatibility problem is shown in Table II.

Taking into account the considerations above our final conclusion was that the Neo4j graph database is a technology that is more well suited to the subject of the compatibility problem.

\section{CTS database}

The CTS database is the core of CTS. The CTS prototype that we have developed is based on the Neo4j graph database. The architecture of the CTS database contains two major domains:

- Nodes and relations space; 


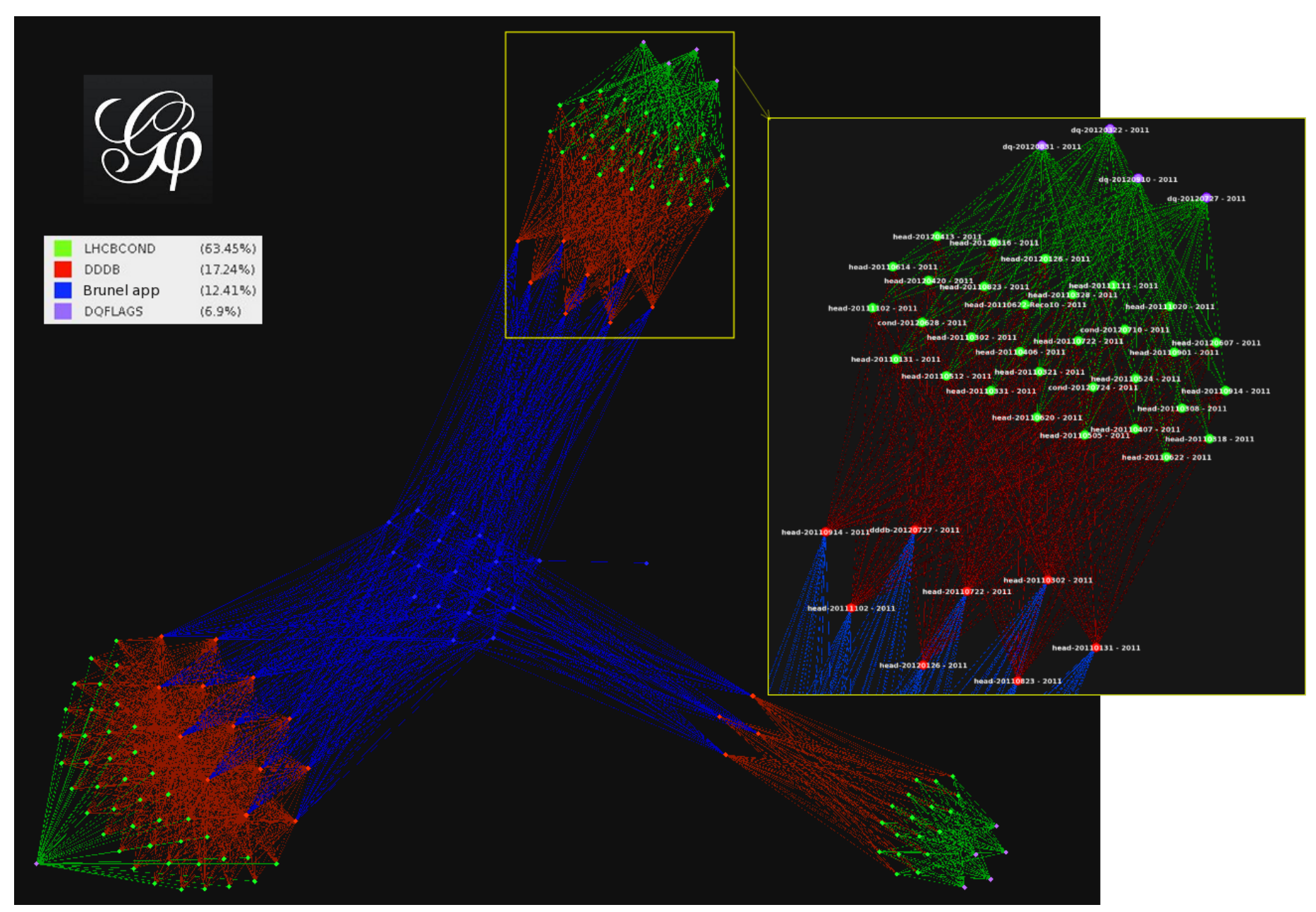

Fig. 5. Real compatibility graph of the LHCb Brunel application (blue) and CondDB states (green, red and purple) is shown. One can clearly see the graphs natural clustering by year of functioning of the experiment. The graph is visualized using Gephi - an open source interactive graph visualization and exploration platform [10].

- Index space.

The first domain contains the actual compatibility information: the application and the CondDB tag nodes, as well as relations between them. The second space is used for indexing the graph nodes in order to perform fast search of the node and relations space. A typical payload of the CTS database is shown in Figure 5. In particular, a compatibility graph between CondDB and $\mathrm{LHCb}$ physics data reconstruction application is shown. Currently it contains 145 nodes, 288 node properties and 1553 node relationships.

\section{E. CTS management}

The CTS management narrows to management of the CTS database content described in the previous section. In the $\mathrm{LHCb}$ experiment this task is performed by the CondDB manager and is, in essence, a process of collecting application and CondDB compatibility relations from the LHCb software developers and keeping the CTS database up-to-date according to that knowledge. Two ways of the CTS database management are available:

- CTS Python command line administration scripts (developed specifically for the LHCb environment, integrated with the CondDB management tools):

- build on developed CTS Python interface based on Py2neo [11] module which interacts in a RESTful style with our CTS Neo4j Server;

- perform automatic injection of new compatibility data into the CTS database;

- perform injection of a complete ground-state graph containing all compatibility entities currently known to the $\mathrm{LHCb}$ experiment.

- Neo4j Server Web Administration interface (is a native Neo4j administration tool):

- monitor the Neo4j Server;

- manipulate and explore graph data;

- interact with the database via various command line consoles (Cypher [12], Gremlin [13] and HTTP);

- view raw data management objects (based on JMX MBeans).

Figures 6, 7, 8, 9 show the snapshots of the Neo4j Web Administration interface. 


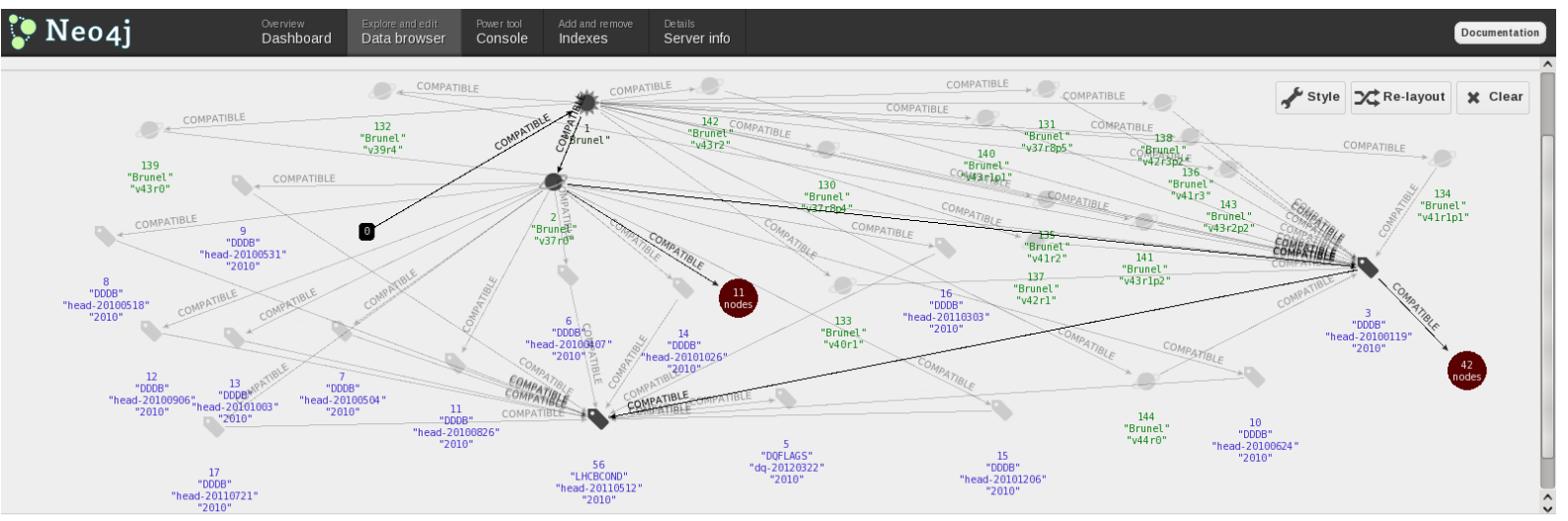

Fig. 6. The Data tab provides an interactive environment to browse, add or modify nodes, relationships and their properties.

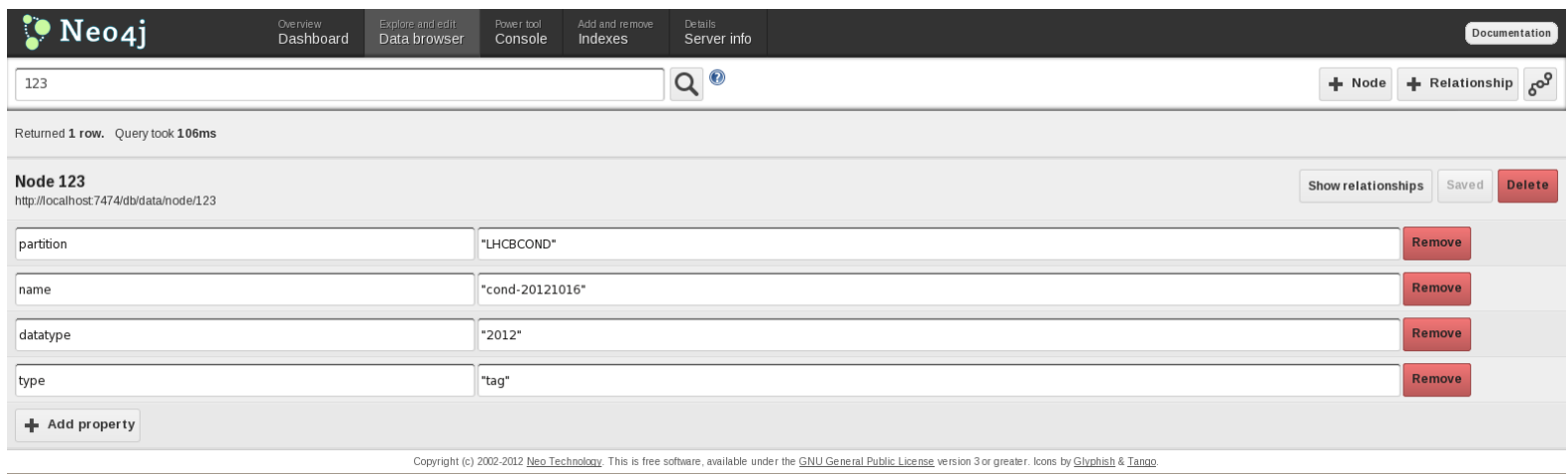

Fig. 7. The Data tab provides an interactive environment to browse, add or modify nodes, relationships and their properties.

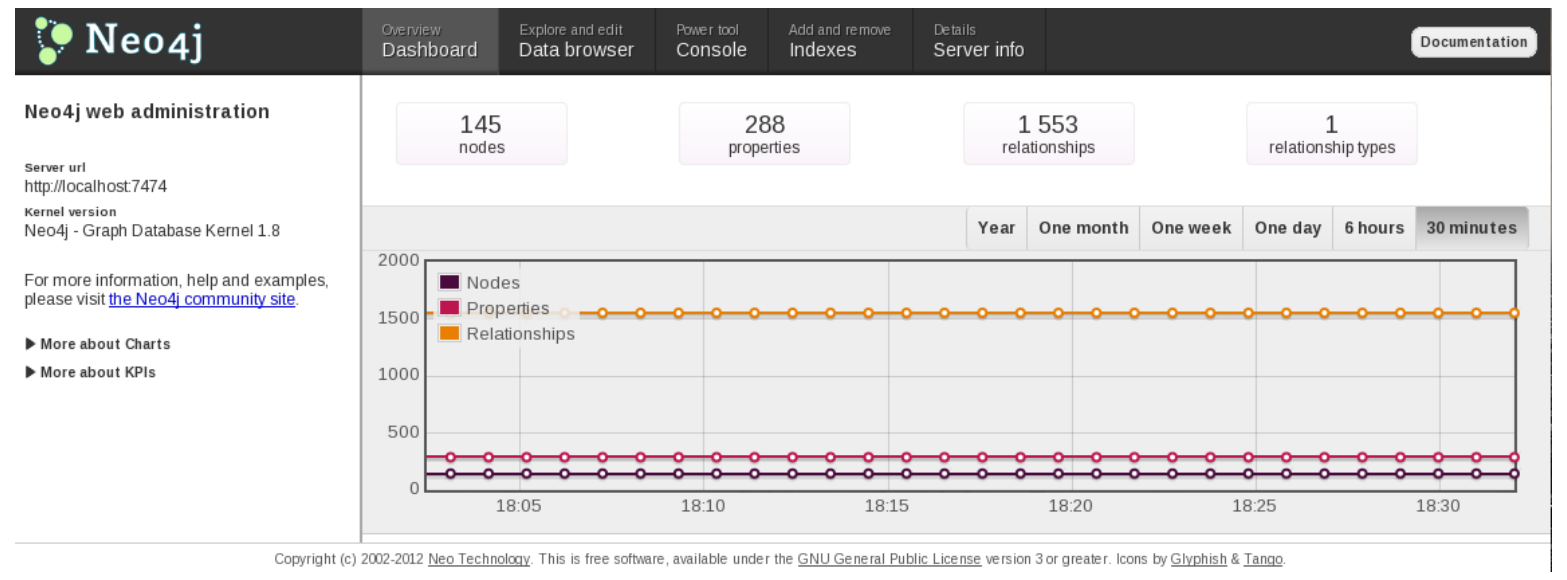

Fig. 8. The Dashboard tab provides an overview of a running Neo4j instance.

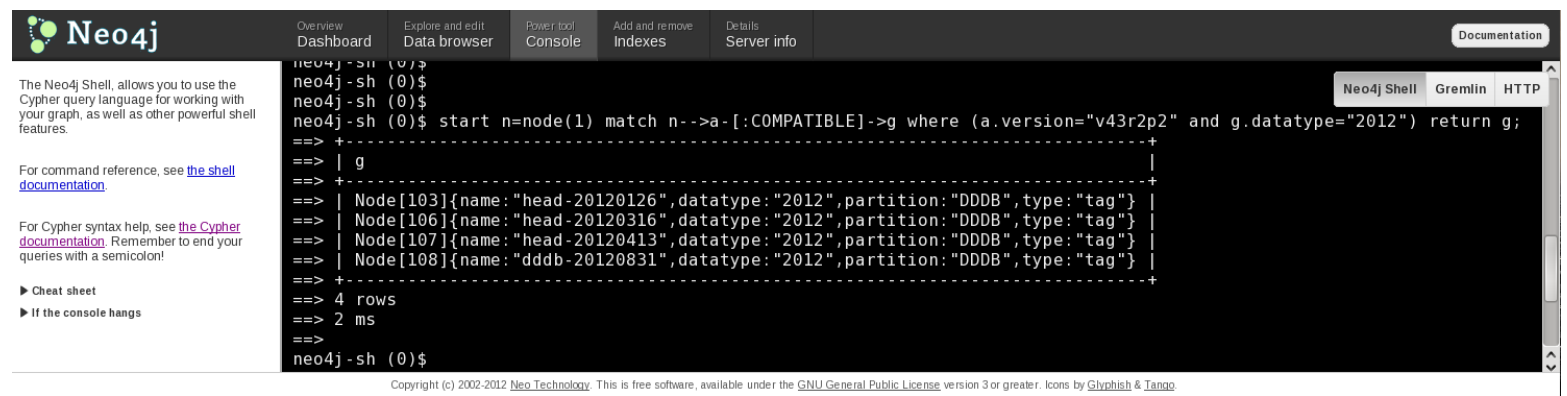

Fig. 9. The console tab provides scripting access to the database via the Gremlin scripting engine, query access via Cypher, and HTTP access via the HTTP console. 


\section{CONCLUSION}

In this paper we described the essence of the compatibility problem faced by the LHCb computing model and proposed a generic way of tracking the compatibility relations between the LHCb applications and the CondDB entities. This approach is based on the graph-theory vision of the problem. In particular, we developed a prototype of the compatibility tracking system with a graph as a basic compatibility information tracking unit which allows to store and access all needed relations elegantly and efficiently. We described the motivation lying behind the choice of the graph-oriented database as the underlying technology for the CTS core. The graph of current crosscompatibilities between $\mathrm{LHCb}$ physics data reconstruction application and CondDB has been also presented being one of the most interesting results of the work. Also the issues of CTS management have been pointed out.

\section{REFERENCES}

[1] LHCb Collaboration, The LHCb Detector at the LHC, JINST 3 (2008) S08005.

[2] LHCb Computing: Technical Design Report, LHCb Collaboration, CERN-LHCC-2005-019, LHCb-TDR-11, CERN, Geneva, 117 p., 2005.

[3] M. Clemencic, N. Gilardi, J. Palacios, LHCb Conditions Database, CERN-LHCb-2006-017, 15th International Conference on Computing In High Energy and Nuclear Physics, Mumbai, India, pp. 347-350, 2006.

[4] E. Yourdon and L.L. Constantine, Structured Design: Fundamentals of a Discipline of Computer Program and Systems Design, Yourdon Press, 1979.

[5] SQLite database engine, Available: http://www.sqlite.org

[6] Neo4j NOSQL graph database project, Available: http://neo4j.org

[7] C. Ireland, D. Bowers, M. Newton, K. Waugh, A Classification of ObjectRelational Impedance Mismatch, DBKDA '09 Proceedings of the 2009 First International Conference on Advances in Databases, Knowledge, and Data Applications, pp. 36-43, 2009.

[8] T. Neward, "The Vietnam of Computer Science", Available: http://blogs. tedneward.com/2006/06/26/The+Vietnam+Of+Computer+Science.aspx, 2007

[9] C. Vicknair et al, A Comparison of a Graph Database and a Relational Database, ACM SE '10 Proceedings of the 48th Annual Southeast Regional Conference, New York, USA, 2010.

[10] Gephi - an interactive graph visualization and exploration platform, Available: https://gephi.org

[11] Py2neo Python library, Available: http://py2neo.org

[12] Cypher - a declarative graph query language, Available: http://docs. neo4j.org/chunked/stable/cypher-query-lang.html

[13] Gremlin - a Turing-complete graph-based programming language, Available: http://gremlin.tinkerpop.com 